\title{
JCEJ Outstanding Paper Award of 2010
}

\author{
Eiji IRITANI ${ }^{1}$ and Yoshiyuki YAMASHITA ${ }^{2}$ \\ ${ }^{1}$ Chairman, Outstanding Paper Awards Subcommittee 2010 of JCEJ, \\ Department of Chemical Engineering, Nagoya University, \\ Furo-cho, Chikusa-ku, Nagoya-shi, Aichi 464-8603, Japan \\ ${ }^{2}$ Editor-in Chief of JCEJ in 2010 , \\ Department of Chemical Engineering, \\ Tokyo University of Agriculture and Technology, \\ 2-24-16, Naka-cho, Koganei-shi, Tokyo 184-8588, Japan
}

Keywords: JCEJ Paper Award, Outstanding Paper

\begin{abstract}
Outstanding Paper Awards Subcommittee of Journal of Chemical Engineering of Japan has assessed the 147 papers published in volume 43 into 2010, and the editorial board finally selected the four papers for JCEJ Outstanding Paper Awards of 2010; those are the papers on "Application of a Microreactor in the Oxidative Dehydrogenation of Propane to Propylene on Calcium Hydroxyapatite and Magnesium ortho-Vanadate Doped and Undoped with Palladium," “On-Line Property Prediction for Industrial Slurry High-Density Polyethylene Processes with Various Grade Transition Modes," "Development of Dimethyl Ether Production Process Based on Biomass Gasification by Using Mixed-Integer Nonlinear Programming," and "Direct Simulation Model of Concentrated Particulate Flow in Pressure-Driven Dead-End Microfiltration."
\end{abstract}

JCEJ Paper Award is given for outstanding contribution to chemical engineering documented in a paper published in Journal of Chemical Engineering of Japan. The selection is made by means of a three-stage process consisting of nomination, first round elimination, and point rating. Outstanding Paper Awards Subcommittee of the journal has assessed the 147 papers published in 2010 , corresponding to volume 43 . The subcommittee selected the candidates of the award and the members of the editorial board confirmed the selection. As a result of this process, the editorial board finally selected the following four papers for the award of 2010.

1. "Application of a Microreactor in the Oxidative Dehydrogenation of Propane to Propylene on Calcium Hydroxyapatite and Magnesium orthoVanadate Doped and Undoped with Palladium" by Shigeru Sugiyama, Naoto Sugimoto, Adusa Ozaki, Yukimi Furukawa, Keizo Nakagawa and Ken-Ichiro Sotowa, The University of Tokushima (Sugiyama et al., 2010)

Citation: This paper demonstrated that a deep oxidation of propylene to $\mathrm{CO}$ and $\mathrm{CO}_{2}$ can be suppressed by employing a microreactor in the oxidative dehydrogenation of propane to propylene, and that the selectivity to propylene is improved remarkably by selecting an appro-

Received on June 13, 2011; accepted on June 14, 2011

Correspondence concerning this article should be addressed to

E. Iritani (E-mail address: iritani@nuce.nagoya-u.ac.jp). priate condition, in comparison with that using a fixedbed continuous-flow reactor. This method proposed by the authors is a novel and effective method to produce propylene which is an important raw material in the petrochemical industry. In addition, by investigating the effects of various factors such as types of catalyst and reaction temperatures on the selectivity in detail, many important knowledegs for process design and determination of optimum process conditions with a microreactor were provided. Therefore, this paper was selected as an outstanding paper.

2. "On-Line Property Prediction for Industrial Slurry High-Density Polyethylene Processes with Various Grade Transition Modes," by Il Kang, Young Kim and Sunwon Park, Korea Advanced Institute of Science and Technology, Honam Petrochemical Corp. and Korea Institute of Machinery and Materials (Kang et al., 2010)

Citation: Reduction of the amount of off-specification products due to frequent grade transitions in polymer production process greatly concerns the petrochemical industry. The authors successfully developed staged property models to predict the melt index and the density of polymers during continuous, discontinuous, and semi-continuous grade transitions. Furthermore, model parameters can be updated on a real-time basis by employing the linear estimation method and the partial property assumption. The practicability of the developed models is clearly demonstrated by applying to an indus- 
trial slurry high-density polyethylene process achieving a $50 \%$ improvement. Thus this paper deserves the Outstanding Paper Award.

3. "Development of Dimethyl Ether Production Process Based on Biomass Gasification by Using Mixed-Integer Nonlinear Programming," by Hosoo Kim, Kyusang Han and En Sup Yoon, Seoul National University (Kim et al., 2010)

Citation: This paper investigates a design of the dimethyl ether (DME) production process involving the use of biomass as a feedstock. First, the superstructure of the DME production process is introduced. Next, a mixedinteger nonlinear programming (MINLP) model is proposed to solve the design problem associated with the selection of technologies and connectivity restrictions. The proposed optimization method provides a novel useful design technique for DME production from biomass feedstock. Thus this paper deserves the Outstanding Paper Award.

\section{4. "Direct Simulation Model of Concentrated Particulate Flow in Pressure-Driven Dead-End Microfiltration" by Tsutomu Ando, Kazuki Akamatsu, Masahiro Fujita and Shin-ichi Nakao, The University of Tokyo and Kogakuin University (Ando et al., 2010)}

Citation: This paper develops a three-dimensional direct numerical simulation model for studying pressure-driven dead-end microfiltration. The numerical model predicts the particle dynamics and fluid flow in a membrane containing straight pores. The authors have demonstrated that their numerical method accurately predicts the deadend filtration process. Furthermore, the authors' method enables one to further understand fouling processes and backwashing mechanisms. For example, the starting point of fouling approaches the feed side of the membrane as the particle concentration increases. The backwash enhances the removal of deposited particles. The computational method developed by the authors not only enables one to further understand dead-end microfiltration, but the new method also contributes to the general field of particulate flows in porous membranes. The impact of this paper on enabling further discovery in the field of porous membranes merits the Outstanding Paper Award.

Congratulations to all the award winners. Winners present their awarded work at the 43th Autumn Meeting of the Society of Chemical Engineers, Japan.

We would also like to acknowledge the cooperation of the Editors and the time and effort of the subcommittee members.

\section{Literature Cited}

Ando, T., K. Akamatsu, M. Fujita and S. Nakao; "Direct Simulation Model of Concentrated Particulate Flow in Pressure-Driven DeadEnd Microfiltration,” J. Chem. Eng. Japan, 43, 815-828 (2010)

Kang, I., Y. Kim and S. Park; "On-Line Property Prediction for Industrial Slurry High-Density Polyethylene Processes with Various Grade Transition Modes," J. Chem. Eng. Japan, 43, 581-594 (2010)

Kim, H., K. Han and E. Yoon; "Development of Dimethyl Ether Production Process Based on Biomass Gasification by Using MixedInteger Nonlinear Programming," J. Chem. Eng. Japan, 43, 671$681(2010)$

Sugiyama, S., N. Sugimoto, A. Ozaki, Y. Furukawa, K. Nakagawa and K. Sotowa; "Application of a Microreactor in the Oxidative Dehydrogenation of Propane to Propylene on Calcium Hydroxyapatite and Magnesium ortho-Vanadate Doped and Undoped with Palladium," J. Chem. Eng. Japan, 43, 575-580 (2010) 